\title{
Soft and Flexible Antennas on Permittivity Adjustable PDMS Substrates
}

\author{
Jovanche Trajkovikj, Jean-François Zürcher, and Anja K Skrivervik \\ Laboratoire d'Electromagnétisme et d'Acoustique, Ecole Polytechnique Fédérale de Lausanne, Station 11, \\ CH-1015 Lausanne, Switzerland \\ jovanche.trajkovikj@epfl.ch
}

\begin{abstract}
This work presents novel techniques for producing substrates for flexible antennas. The technique we propose is based on the use of an already existing and widely used substrate material Polydimethylsiloxane (PDMS), where the dielectric properties of the substrate are adjusted by loading the PDMS with low or high permittivity inclusions. The low adhesion characteristics of PDMS are overcome by immersing the conducting parts of the antenna inside the substrate, at the same time sealing the antenna against the influence of dust, or water. A patch antenna prototype is realized and characterized. The built antenna is soft and flexible and it shows good radiation characteristics in terms of input matching and total gain. Measurements are in a good compliance with the simulation results.
\end{abstract}

Keywords-flexible electronics, flexible substrates, soft and flexible antennas, PDMS, polymers, patch antenna, robustness, wearable antennas

\section{INTRODUCTION}

In recent years, flexible electronics have received an increasing attention due to their wide use in different types of applications. Flexible displays, smart tags and wearable products are just few examples where they find their use [1]. They are particularly interesting for medical applications, where they are widely deployed. An example is presented in [2], where the neural prosthetics interface is based on a flexible electronics using flexible substrates.

Following the same trends, antennas used in such electronic systems should also be flexible, thus new solutions are required. One of the main challenges while designing flexible antennas is to find adequate substrate materials. Flexible, also known as stretchable, antennas find their use both in implantable [2] and wearable applications [3], where medical applications is the largest field.

Among the variety of polymer materials (e.g., Liquid Crystal Polymers (LCP), Polyetherimide (PEI) or Polyethylene terephthalate $(\mathrm{PET})$ ) [1], proposed in the literature, PDMS is one of the most frequently used substrate material for flexible antennas. For instance, Liu et al. [3], designed a stretchable Planar Inverted F Antenna (PIFA) and Dipole antenna, using PDMS as a substrate. Moulder et al. [4], introduced PDMS antennas for microwave and millimeter wave systems, while Hage-Ali et al. [5] show a millimeter-wave patch array antenna printed on PDMS. Hence, this substrate has become a widely used material for modern flexible electronics.
This trend can be widely explained by PDMS' characteristics: it is chemically inert, thermally stable, permeable to gases, simple to handle and manipulate, and exhibits isotropic and homogenous properties [6]. It is an excellent water isolator thus enabling a long-term, stable device (e.g. antenna) operation. PDMS has good RF and mechanical characteristics, a low dielectric constant and a good chemical stability. The initial fluid state of the PDMS allows preparation of composite substrates of various concentrations, thus controlling the range of dielectric constants that can be obtained. Moreover, the fluid state of the elastomer also offers control over the substrate thickness as well as the possibility of immersing the antenna inside the substrate [2].

Beside all the benefits of using PDMS as an antenna substrate, some challenges have to be met. Metal printing is one of the main issues to be solved. Several processes have been proposed and presented in [6], in order to overcome the low adhesion problem.

In this paper we propose a new method to design antennas using PDMS. In the first part we developed an idea on how to modify the dielectric permittivity of the substrate by adding inclusions to the PDMS. We concentrate our efforts on lowering the permittivity, in order to enhance the radiation characteristics (bandwidth/efficiency) of the antenna (see for instance [7]). In the second part we propose to integrate substrate and conductive parts, in order to manufacture flexible and sealed antennas. To this aim, copper mesh sheets will be used for the conductive parts, keeping thus the antenna flexible. The results will be compared to the use of standard copperberyllium sheets for the conductive parts of the antenna. Finally, the paper presents a patch antenna prototype constructed using the proposed technique, comparing the simulated and measured results

\section{ANTENNA CONCEPT}

In this section we present first how to obtain a dielectric substrate having the appropriate dielectric permittivity, and then the different types of conducting sheets that can be used to manufacture the antennas.

\section{A. Dielectric part}

Considering the initial liquid state of PDMS (Silgard-184 kit) [8] additional materials can be added to the mixture in the phase of preparation. The idea is that the added materials 
should have a relative electric permittivity lower than PDMS itself, thus resulting in a lower final permittivity. Moreover the losses should be low.

Several experiments were done using different additive materials: phenolic, silicate and hollow glass microspheres [8]. After comparing the measurements, the best results were obtained by using hollow glass microspheres. The microspheres have a diameter between 20 and $50 \mu \mathrm{m}$, and as they are filled by air, a relative permittivity close to 1 .

The measured results for the relative electric permittivity of the produced pure PDMS and the PDMS with the introduced hollow glass microspheres are presented in Fig.1. The weight density of the glass microspheres with the respect to the Silgard-184 RTV weight is $12.7 \%$. First measurements have been performed using the waveguide (WG) method (dielectric inside a waveguide, reflection/transmission measurement) in X-band, between 9.46-11.56 GHz. Comparing our measurements for pure PDMS to the results presented in literature [9] (relative electric permittivity is $2.77 \pm 0.05$ across the $0.1-40 \mathrm{GHz}$ range) our values $(\approx 2.69)$ are in a good compliance. The small discrepancies appear due to the use of different Silgard kits, 184 in our case, and 182 in the presented paper [9]. Since we were generically interested in the dielectric properties of PDMS with the glass microspheres at $2.45 \mathrm{GHz}$, an additional measurement has been made at low frequencies $(200 \mathrm{MHz}-5 \mathrm{GHz})$ using an APC-7 probe and the Agilent 85070 measurement facility. The results of the measurements from both methods are shown in Table 1.

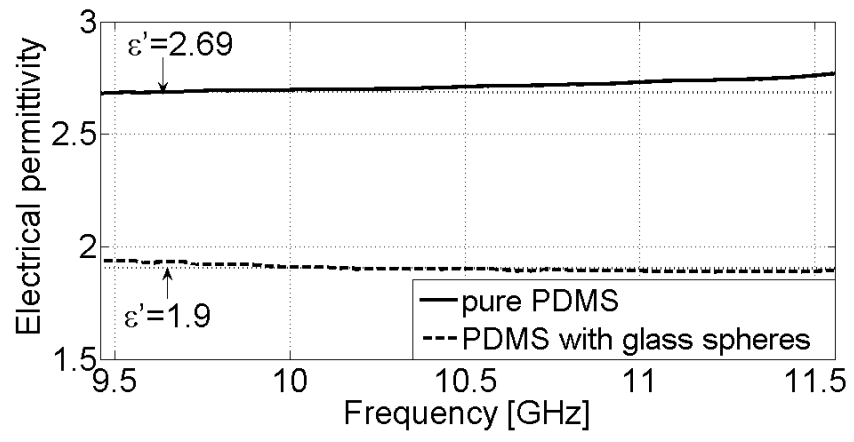

Figure 1. Measuremnet results for the electric permittivity for pure PDMS and PDMS with glass microspheres using the WG method

TABLE I. MEASUREMENT RESULTS

\begin{tabular}{|c|c|c|}
\hline PDMS with glass microspheres & $\varepsilon^{\prime}$ & $\tan \delta$ \\
\hline WG method $(9.46-11.56 \mathrm{GHz})$ & 1.9 & 0.014 \\
\hline Probe method $(200 \mathrm{MHz}-5 \mathrm{GHz})$ & 2.0 & 0.03 \\
\hline
\end{tabular}

Since the complete process is an in-house custom made performance, some uncertainties are present. Due to the hand mixing of the PDMS and the glass microspheres it is possible that a certain percentage of the spheres is broken, resulting in a different permittivity than expected. Also in some of the final products, air bubbles appeared due to the non-perfect degassing vacuum process. The degassing process was performed in a special plastic bowl, connected to a vacuum pump through plastic pipes. Once the conductive parts of the antenna are placed in the mold, and the latter filled with the PDMS substrate, everything is enclosed in the bowl. The pump extracts the air through the pipes, while the pressure level is controlled with the manometer connected to the one of the pipes. The complete setup is shown in Fig. 2 a).

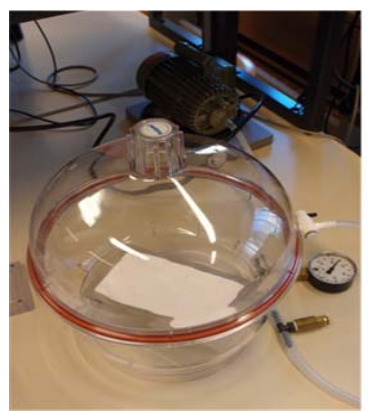

a)

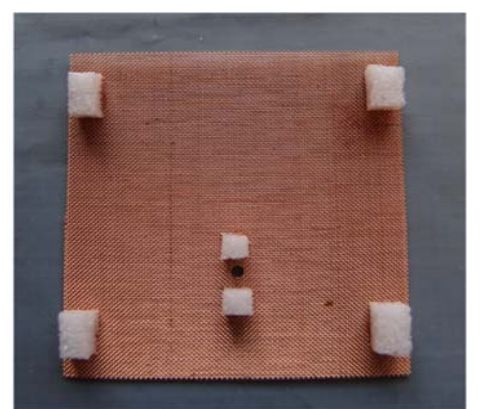

b)
Figure 2. a) Vaccum setup, b) Copper mesh sheet (patch) with the glued foam spacers for maintaing constant distance between the groundplane and the patch

With the proposed way of mixing PDMS substrate with enclosures we managed to reduce the overall relative electric permittivity of PDMS from 2.7 to 1.9.

\section{B. Conductive part}

As presented in the introduction, there are several techniques for attaching metallization layers on a PDMS substrate [3], [6]. These techniques are based on complex chemical and micromachining methods, requiring adequate equipment and conditions. Using the facilities we have in our laboratory, we tried to develop simple methods that can be applied for in-house customized design of flexible antennas.

In a first approach we tried to integrate/attach a copperberyllium $(\mathrm{Cu}-\mathrm{Be})$ conductive plate to the PDMS substrate. The whole process of integrating/attaching was performed while the PDMS was in the initial liquid state. After the curing, once the final antenna was ready, we tried to assess the antenna's mechanical properties, like bending and twisting. As it can be seen from Fig. 3 a), the copper-beryllium plate detached itself from the PDMS substrate, first on the corners then over the entire structure. Hence, it was decided to use another approach in order to obtain better adhesion characteristics.

In the second experiment we used copper mesh sheets [10], Fig. 2 b) as a conductive material. The main advantage over the $\mathrm{Cu}-\mathrm{Be}$ plates is their intertwined structure, which allows easy penetration of the PDMS material through the mesh, and better preserves the flexibility of the structure. Again, as in the previous case, the attaching of the two materials was performed in the initial phase of the preparation, while the PDMS is still in a liquid state. Since the PDMS completely surrounds the mesh, the latter is completely encapsulated in the substrate material, Fig. 3 b). This completely seals the antenna. The complete process is explained in section III, antenna design. 
PDMS in a combination with copper mesh conductors performs excellent results in terms of adhesion, at the same time making the structure flexible and robust to bending and twisting.

For design purposes we have used a pure copper mesh with $110 \mu \mathrm{m}$ diameter wires and $140 \times 140 \mu \mathrm{m}$ apertures for the conductive parts [10]. Fig. 3 b) shows the cross-section of the final product, the mesh "sandwiched" inside the PDMS substrate.

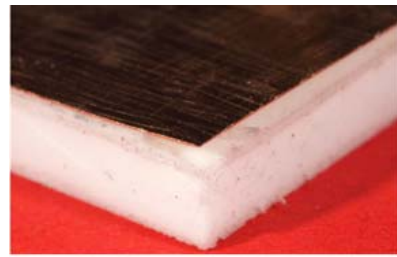

a)

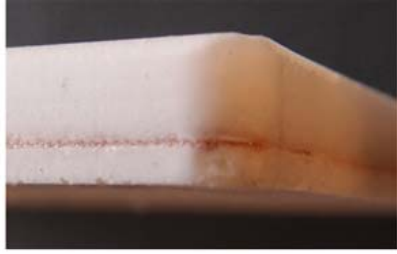

b)
Figure 3. PDMS cross-sections: a) Copper-Berylium plate attached to the PDMS - poor adhesion, b) Copper mesh sheet "sandwiched" between the PDMS layers - excellent adhesion

\section{Antenna Design, Simulations Measurements AND CHARACTERIZATION}

A simple patch antenna operating at $2.45 \mathrm{GHz}$ is considered as an example in order to show the appropriateness of PDMS based substrates for flexible antennas. The antenna is designed and simulated in a commercially available 3D electromagnetic software, HFSS [11]. After obtaining the optimal performance of the simulated antenna, a prototype was built.

The conductive parts of the antenna, i.e. the patch and the ground plane where cut to the right dimensions and the SMA pin soldered in place. They were then placed in a mold, using tiny foam spacers beneath the ground plane and between the ground plane and the patch, to ensure the right distance between these layers. Two O-rings were used in order to seal the connector and to prevent the leakage of PDMS through the bottom hole (for the SMA connector) in the mold. The mold was than filled with the prepared substrate mixture (PDMS + glass spheres), as shown in Fig. 5 a). After completing the filling process the mold was covered from the top side and placed in an oven at $60^{\circ} \mathrm{C}$ for curing.

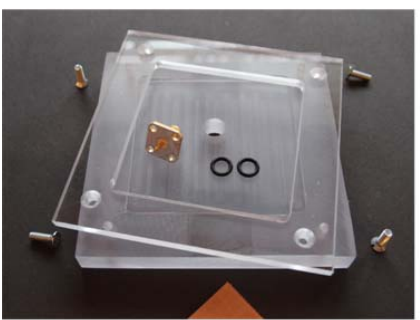

a)

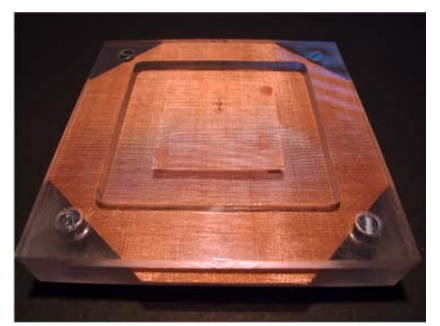

b)
Figure 4. a) In-house designed mold made of polyvinyl chloride (PVC) plastic, b) Assembled mold with the conductive antenna parts in place, ready to be filled with the liquid (PDMS + glass microspheres) material

The finished antenna has a size of $45 \times 45 \mathrm{~mm}$; the connector is attached from the bottom side, and as it can be seen from the
Fig. 5 b), the conductive parts of the antenna are fully encapsulated inside the PDMS substrate.

The antenna was measured and characterized with a vector network analyzer and in an anechoic chamber. The measured results are in a good compliance with the simulations. The antenna has a good input matching, Fig. 6, and a symmetric radiation pattern, E plane, Fig. 7, H plane Fig. 8.

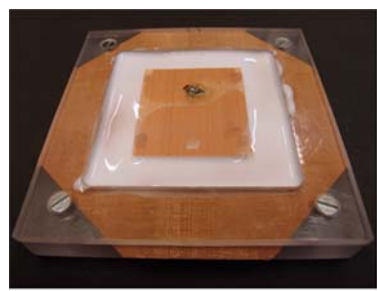

a)

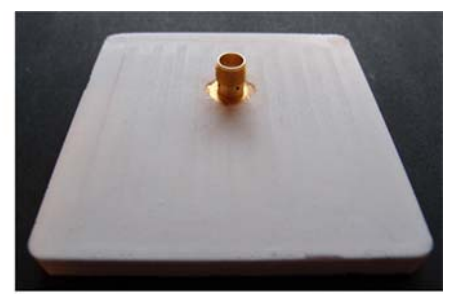

b)
Figure 5. a) Filling the antenna mold with PDMS (visible PDMS penetration through the copper mesh), b) Soft and flexible antenna (bottom side) ready for measuremnts and use

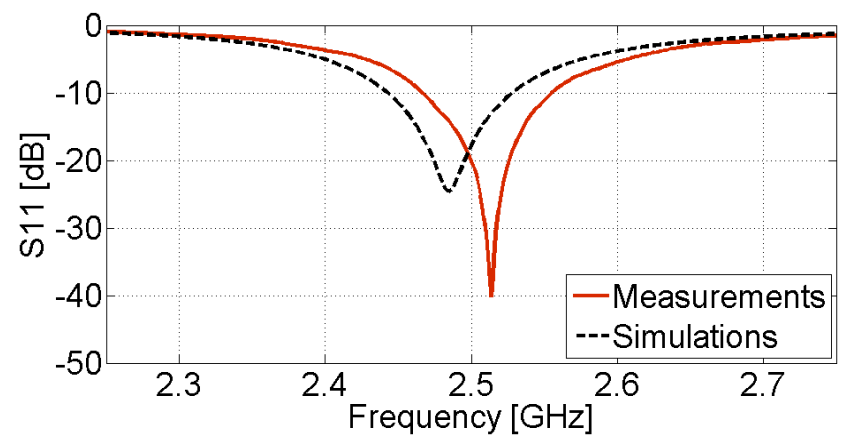

Figure 6. Input reflection coefficient

The antenna is supposed to be completely waterproof (except the SMA connector, which is a standard one). After protecting the SMA connector with an adequate cap, the antenna has been put inside a washing machine (at $60^{\circ}$ ) for a complete cycle. At the end of the operation the antenna looked exactly as before. The antenna was re-measured again and no significant discrepancies were observed, showing the good sealing performances of this technology.

Introducing a new way of preparing substrates in a combination with proper conductive sheets, we were able to produce a sustainable soft and flexible antenna. A built antenna prototype has excellent results in terms of radiation characteristics, input matching as well as mechanical properties. Moreover the antenna is waterproof.

\section{CONCLUSIONS}

New techniques for preparing substrates for soft and flexible antennas have been presented. Control over the substrate dielectric properties has been obtained by loading PDMS with low permittivity inclusions. In order to circumvent the low adhesion characteristic of PDMS, a copper mesh sheet was used as a conductive material. The intertwined structure of the mesh sheets allows an easy integration with the PDMS and its penetration through the mesh, thus making the antenna 
flexible and completely sealed, protecting it from external influence (water, humid, dust, etc.) and making it robust against mechanical exposures (twisting, bending, etc.).

Finally, a single patch antenna was built in order to illustrate the technology, which can of course easily be used for more complex antennas. The built antenna responded to the initial requirements. It is soft and flexible, completely sealed, and has good radiation characteristics, where the simulations and measurements are in a good compliance.

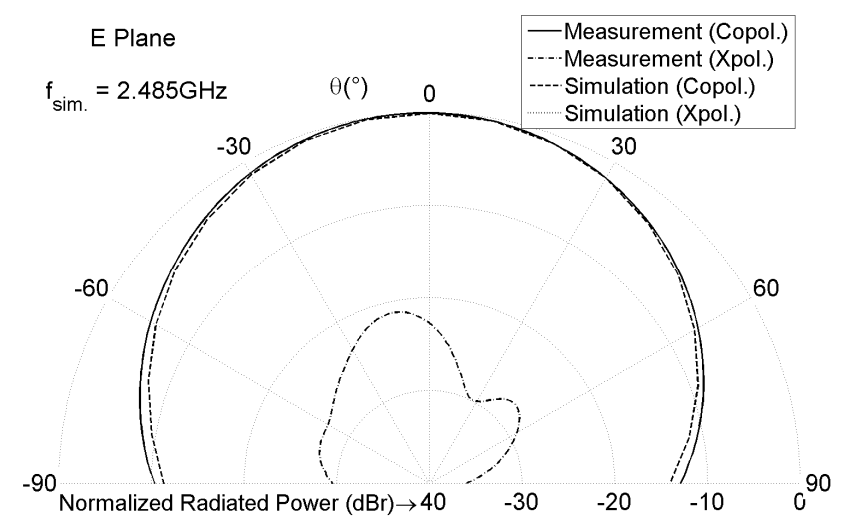

Figure 7. Measured and simulated far-field radiation pattern, E plane

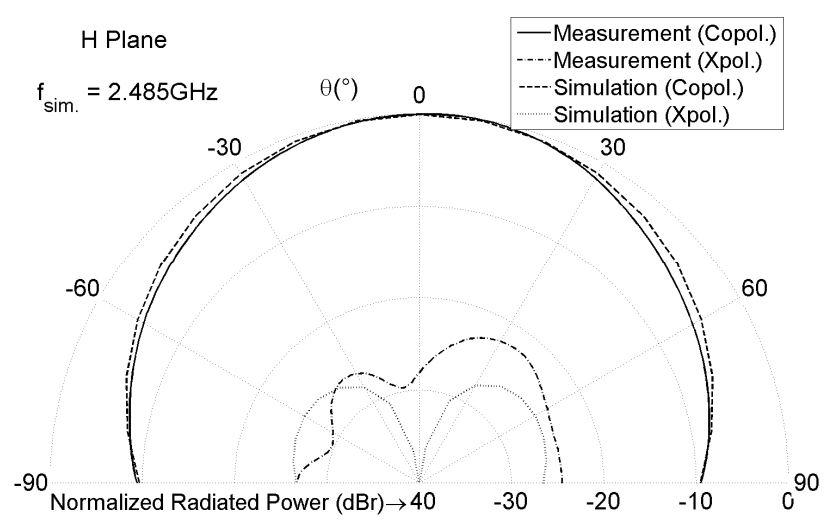

Figure 8. Measured and simulated far-field radiation pattern, $\mathrm{H}$ plane

\section{REFERENCES}

[1] C.-P. Lin, C.-H. Chang, Y. T. Cheng, and C. F. Jou, "Development of a Flexible SU-8/PDMS-Based Antenna," Antennas and Wireless Propagation letters, vol. 10, pp. 1108-1111, 2011.

[2] E. Apaydin, "Microfabrication Techniques for Printing on PDMS Elastomers for Antenna and Biomedical Applications," Ohio State University, 2009.

[3] Q. Liu, K. L. Ford, R. Langley, A. Robinson, and S. Lacour "Stretchable Antennas," in European Conference on Antennas and Propagation, 2012, pp. 10-13.

[4] W. Moulder, Y. Zhou, E. Apaydin, L. Dai, R. Emrick, and P. I. J. L. Volakis, "Polymer Based Antennas for Next Generation Microwave and Millimeter Wave Systems Motivation \& Goals," 2009.

[5] P. Hage-Ali, S.; Tiercelin, N.; Coquet, P.; Sauleau, R.; Preobrazhensky, V.; Pernod, "Millimeter-wave patch array antenna on ultra flexible micromachined Polydimethylsiloxane (PDMS) substrate," in IEEE Antennas and Propagation Society International Symposium, 2009. APSURSI '09., 2010, no. 1, pp. 1-4.

[6] A. Mata, A. J. Fleischman, and S. Roy, "Characterization of Polydimethylsiloxane (PDMS) Properties for Biomedical Micro/Nanosystems," Biomedical Microdevices, vol. 7, no. 4, pp. 281-293, Dec. 2005.

C. A. Balanis, Antenna Theory Analisys and Design, vol. 28, no. 3. 2005, p. 1117.

[8] F. Tel, "Faserverbundwerkstoffe Matériaux composites Composite materials," 2011. [Online]. Available: swiss-composite.ch.

[9] N. J. Farcich, S. Member, J. Salonen, and P. M. Asbeck, "SingleLength Method Used to Determine the Dielectric Constant of Polydimethylsiloxane," IEEE Transactions on Microwave Theory and techniques, vol. 56, no. 12, pp. 2963-2971, 2008.

[10] "100 Mesh Copper .0045" Wire Dia. 36 Inches Wide,” 2011. [Online]. Available: www.twpinc.com. 